COMUNICAÇÃO CIENTÍFICA

\title{
AÇÃO DE DIFERENTES PREPARAÇÕES DE EXTRATO PIROLENHOSO SOBRE Brevipalpus phoenicis (GEIJSKES) ${ }^{1}$
}

\author{
MARIANGELAALVES ${ }^{2}$, JAIRO OSVALDO CAZETTA $^{3}$, MARIAANDRÉIANUNES $^{5}$, CARLOS AMADEU LEITE DE OLIVEIRA $^{6}$, \\ CARLOSALEXANDRECOLOMBI ${ }^{7}$
}

RESUMO - O objetivo deste trabalho foi avaliar a forma de ação de duas preparações de extrato pirolenhoso aplicadas diretamente sobre Brevipalpus phoenicis, que é o ácaro vetor da leprose dos citros, um dos principais problemas da citricultura Paulista. Para o experimento, foram utilizados ácaros adultos mantidos numa criação-estoque no laboratório de Acarologia da UNESP - Universidade Estadual Paulista, em Jaboticabal-SP. Os tratamentos foram constituídos por duas diferentes preparações (destilado e decantado) de extrato pirolenhoso de eucalipto nas proporções EP:água de 1:600; 1:300 (normalmente recomendadas); 1:150; 1:75; 1:38; 1:19 e de água (testemunha), com 7 repetições. Cada parcela foi constituída de 10 ácaros mantidos sobre um fruto de laranja, em arena de 2,5 cm de diâmetro, delimitada com cola adesiva tipo Tanglefoot ${ }^{\circledR}$. As aplicações foram efetuadas em Torre de Potter, pulverizando-se $2 \mathrm{~mL}$ por fruto das soluções correspondentes aos diferentes tratamentos. Os frutos foram mantidos em sala climatizada a $27 \pm 1^{\circ} \mathrm{C}$, e as avaliações foram realizadas 24 e 48 horas após a aplicação dos tratamentos, determinando-se o número de ácaros mortos (mortalidade) e retidos na barreira adesiva (repelência). Os dois tipos de extrato pirolenhoso testados não apresentaram repelência significativa sobre Brevipalpus phoenicis; ambos induziram mortalidade significativa somente para concentrações acima de 1:150, com efeito mais pronunciado para o destilado; há um aumento na mortalidade de 24 para 48 horas após a aplicação; a ação protetora preconizada pela aplicação de baixas doses (1:300 a 1:600) nas plantas não é devida à mortalidade e repelência pelo contato direto do extrato pirolenhoso sobre os ácaros. Termos para indexação: ácido pirolenhoso, ácaro, leprose, citros.

\section{ACTION OF DIFFERENT PYROLIGNEOUS EXTRACT PREPARATIONS WHEN APPLIED ON Brevipalpus phoenicis (GEIJSKES)}

\begin{abstract}
The aim of this work was to evaluate the acaricide and repellent effects of two different pyroligneous extract preparations (PE) applied directly on Brevipalpus phoenicis. This mite is the vector or citrus leprosies, which is one of the main problems of São Paulo State citrus culture. This experiment was carried out with mites derived of the colony-supply maintained in the laboratory of Acarology of the Unesp - São Paulo State University, at Jaboticabal. The experiment consisted of application of water (control) and two distinct way of preparations (distilled and decanted) of pyroligneous extract (PE) of eucalyptus in 6 volumetric ratios PE: water of 1:600, 1:300, 1:150, 1:75, 1:38 and 1:19, with 7 replications. Each plot with 10 mites maintained on an orange fruit, in enclosure of $2.5 \mathrm{~cm}$ diameter isolated by Tanglefoot ${ }^{\circledR}$ (The Tanglefoot Company: USA) adhesive barrier. After confection of the enclosures and mites transference, $2 \mathrm{~mL}$ of the solutions corresponding to the different treatments were applied per fruit, by using a Potter's Tower. Then, the fruits were maintained in a room at $27 \pm 1^{\circ} \mathrm{C}$, and evaluations of the number of dead mites (mortality) and restrained in the adhesive barrier (repellence) were carried out 24 and 48 hours after treatment application. The distilled PE presented more mortality in comparison to decanted PE for concentrations higher than 1:150. None of two types of extract showed significant repellence effect on $B$. phoenicis. The protective action recognized for low doses (1:300 to 1:600, normally recommended), is not due to mortality or repellence by direct contact of these products with mites.
\end{abstract}

Index terms: pyroligneous acid, leprosies mite, citrus.

O extrato pirolenhoso, também conhecido como ácido pirolenhoso, líquido pirolenhoso ou vinagre de madeira, é um líquido resultante da condensação da fumaça originada da fabricação de carvão. Segundo Miyasaka et al. (2001), o extrato pirolenhoso bruto não deve ser utilizado na agricultura sem ser purificado para eliminar o alcatrão, que é solúvel logo após a obtenção do produto, o que pode ser realizado industrialmente por destilação sob vácuo ou artesanalmente via decantação, mantendo-se o produto em repouso por tempo superior a 100 dias. Tal repouso promove a separação em três fases: a fase superior, que contém óleos leves, a inferior composta pelo alcatrão, que se precipita durante o período de repouso, e a fase central

${ }^{1}$ (Trabalho 101-06). Recebido em: 21-07-2006. Aceito para publicação em : 14-05-2007.

${ }^{2}$ Bióloga, Mestre em Agronomia [Produção Vegetal], Unesp - Universidade Estadual Paulista, Jaboticabal-SP.

${ }^{3}$ Prof. Adjunto, Unesp - Universidade Estadual Paulista, Departamento de Tecnologia, Via de Acesso Prof. Paulo Donato Castellane, s/n, 14884-900, Jaboticabal-SP. cazetta@fcav.unesp.br.( Autor correspondente).

${ }^{5}$ Bióloga, Doutoranda em Agronomia [Entomologia Agrícola], Unesp - Universidade Estadual Paulista, Jaboticabal-SP.

${ }^{6}$ Prof. Titular, Unesp-Universidade Estadual Paulista, Departamento de Fitossanidade, Jaboticabal-SP.

${ }^{7}$ Engenheiro Agrônomo, Mestrando em Agronomia [Entomologia Agrícola], Unesp - Universidade Estadual Paulista, Jaboticabal-SP. 
que é o extrato pirolenhoso apropriado para ser usado na agricultura, após as diluições indicadas para cada caso.

O extrato pirolenhoso filtrado após a decantação é composto de 80 a $90 \%$ de água e 10 a 20\% de compostos orgânicos, sendo o principal o ácido acético. Quando aplicado em diluições de 300 a 600 vezes, apresenta-se promissor no controle de pragas e doenças, podendo ser aplicado isoladamente ou misturado com outros extratos de plantas (Maekawa, 2002).

Apesar dos efeitos preconizados para o extrato pirolenhoso, existe escassez de informações científicas que possam dar suporte à utilização deste produto e à compreensão dos mecanismos pelos quais funciona, especialmente no que se refere à proteção das plantas contra pragas e doenças de grande importância, como é o caso da leprose do citros, doença transmitida pelo ácaro Brevipalpus phoenicis. Esta doença manifesta-se através de lesões nos frutos, bem como nos ramos e folhas quando em alto nível de infestação (Campos Neto et al., 1993). Seu controle tem sido realizado pela aplicação de pesticidas sintéticos que podem selecionar indivíduos resistentes (Alves et al., 2005), elevam os custos de produção e também não podem ser usados na agricultura orgânica. Neste contexto, a aplicação de produtos, como o extrato pirolenhoso, poderia ser uma boa alternativa para o controle destes ácaros. Levando em conta o acima exposto, foi desenvolvido este trabalho com o objetivo de avaliar a ação (mortalidade ou repelência) de duas formas de preparação do extrato pirolenhoso (produzido e destilado industrialmente ou purificado por decantação) sobre $B$. phoenicis em condições de laboratório, visando a obter informações importantes sobre o modo de ação e o potencial de uso desse extrato no controle de ácaros.

Para a execução deste trabalho, foram utilizadas fêmeas adultas de $B$. phoenicis, obtidas de criações-estoque mantidas em sala climatizada $\left(25 \pm 3^{\circ} \mathrm{C}\right.$ e UR $\left.70 \pm 10 \%\right)$, no laboratório de Acarologia da FCAV/UNESP, em Jaboticabal-SP. Estes ácaros foram mantidos dentro de arenas de 2,5 cm de diâmetro delimitadas por cola adesiva tipo Tanglefoot ${ }^{\mathrm{O}}$ (The Tanglefoot Company: USA), em frutos de laranja.

O extrato pirolenhoso destilado foi obtido da Biocarbo Indústria e Comércio Ltda. e comercializado com o nome de Biopirol $^{\mathrm{O}}$. O extrato pirolenhoso artesanal, oriundo de forno de produção de carvão de eucalipto, foi armazenado em tambor de polietileno, mantido tampado e em repouso por cerca de 100 dias, para a obtenção da fase aquosa.
O delineamento utilizado foi o inteiramente casualizado, no esquema fatorial $2 \times 7 \times 2$ (tipos de EP x proporções EP: $\mathrm{H}_{2} \mathrm{O}$ x tempo de avaliação), totalizando 28 tratamentos e 7 repetições. Os tratamentos testados foram dois tipos de extrato pirolenhoso (destilado e decantado) em seis proporções EP:água, 1:600; 1:300; $1: 150 ; 1: 75 ; 1: 38$ e $1: 19$, correspondendo às porcentagens de 0,17 ; 0,$33 ; 0,67 ; 1,33 ; 2,67$ e $5,33 \%(v / v)$. Como testemunha, utilizou-se água destilada.

O experimento foi montado no mesmo local e condições da criação-estoque, utilizando-se de metodologia já padronizada para avaliar efeito de mortalidade e repelência de defensivos agrícolas. Cada unidade experimental constou de 10 fêmeas adultas, aproximadamente de mesma idade, em um fruto de laranja da variedade Pêra, parafinado e delimitado por arenas de $2,5 \mathrm{~cm}$ de diâmetro, isoladas com barreira adesiva. Após a montagem das arenas e transferência dos ácaros, foram realizadas pulverizações, Torre de Potter, de $2 \mathrm{~mL}$ de cada concentração e os frutos mantidos em sala climatizada $\left(25 \pm 3^{\circ} \mathrm{C}\right.$ e UR $\left.70 \pm 10 \%\right)$, durante o período de condução do experimento. As avaliações foram realizadas 24 e $48 \mathrm{~h}$ após a pulverização, determinando-se o número de ácaros mortos na arena (mortalidade) e o número de ácaros na barreira adesiva, este último como indicativo da repelência dos produtos. Os dados foram submetidos à análise de variância, pelo teste $\mathrm{F}$, e as médias comparadas pelo teste de Tukey, a $5 \%$ de probabilidade.

A mortalidade de B. phoenicis, avaliada $24 \mathrm{~h}$ após a aplicação dos tratamentos, aumentou proporcionalmente à elevação da concentração para ambos os tipos de EP. Comparado com o tratamento-testemunha (aplicação de água), ambos os tipos de EP tiveram efeitos significativos somente a partir da diluição de 1:150, que induziu uma mortalidade ao redor de 11\% (Tabela 1 e Figura 1). Entretanto esta diluição corresponde à concentração acima da normalmente recomendada para aplicação nas plantas, que está entre 1:300 e 1:600 (Maekawa, 2002). Para concentrações ainda maiores, ou seja, nas diluições de 1:75; 1:38 e 1:19, o aumento na mortalidade foi significativo e proporcional à concentração aplicada, sendo que, nestas diluições, o EP destilado diferenciouse do decantado, apresentando mortalidade mais elevada. Nesta avaliação, a máxima mortalidade observada foi para a solução mais concentrada (1:19), que promoveu a mortalidade de $93 \%$ e $41 \%$, respectivamente, para o EP destilado e decantado (Tabela 1 e Figura 1).

Na segunda avaliação, realizada $48 \mathrm{~h}$ após a aplicação dos

TABELA 1 - Efeito da interação entre o tipo de extrato pirolenhoso e as diluições sobre a mortalidade $(\%)$ de $B$. phoenicis $\left(25 \pm 3^{\circ} \mathrm{C}\right.$ e UR $70 \pm 10 \%$ ).

\begin{tabular}{|c|c|c|c|c|c|c|c|c|}
\hline \multirow{2}{*}{ Tipo de EP } & \multicolumn{6}{|c|}{ Proporções EP: $\mathrm{H}_{2} \mathrm{O}$} & \multirow{2}{*}{ Testemunha } & \multirow{2}{*}{ Teste F } \\
\hline & $1: 19$ & $1: 38$ & $1: 75$ & $1: 150$ & $1: 300$ & $1: 600$ & & \\
\hline Destilado & $95,00 \mathrm{Aa}$ & $67,86 \mathrm{Ba}$ & $40,00 \mathrm{Ca}$ & $11,43 \mathrm{Da}$ & $4,29 \mathrm{Ea}$ & $2,14 \mathrm{Ea}$ & $0,71 \mathrm{Ea}$ & $974,27 * *$ \\
\hline Decantado & $49,29 \mathrm{Ab}$ & $28,57 \mathrm{Bb}$ & $11,43 \mathrm{Cb}$ & $10,71 \mathrm{Ca}$ & $5,00 \mathrm{Da}$ & $2,14 \mathrm{Da}$ & $0,71 \mathrm{Da}$ & $216,15^{* *}$ \\
\hline Teste F & $728,95 * *$ & $538,35 * *$ & $284,75^{* *}$ & $0,18^{\mathrm{ns}}$ & $0,18^{\mathrm{ns}}$ & $0,00^{\mathrm{ns}}$ & $0,00^{\mathrm{ns}}$ & \\
\hline
\end{tabular}

Médias seguidas de mesma letra maiúscula na linha e minúscula na coluna não diferem entre si, pelo teste de Tukey, a $5 \%$ de probabilidade.

** significativo ao nível de $1 \%$ de probabilidade.

ns não-significativo

Rev. Bras. Frutic., Jaboticabal - SP, v. 29, n. 2, p. 382-285, Agosto 2007 
tratamentos, verificou-se um aumento de mortalidade em comparação com resultados da avaliação anterior (Tabela 2). Pelos dados apresentados na Figura 2, pode-se estimar que a mortalidade alcançaria $100 \%$ pela aplicação do EP destilado na concentração ao redor de $3,2 \%(\mathrm{v} / \mathrm{v})$, o que corresponderia a uma diluição próxima de 1:30. Nesta segunda avaliação, o EP decantado continuou apresentando valores de mortalidade menores que o destilado (Tabela 2 e Figura 2). O efeito mais pronunciado de mortalidade do EP destilado, em comparação ao decantado, pode ser devido ao provável aumento da concentração de compostos voláteis provocado pela destilação seca utilizada na produção do EP destilado.

A análise da interação entre as diluições de EP e o tempo após a aplicação dos tratamentos revelou um aumento da mortalidade com o tempo para as diluições testadas, exceto 1:600 e testemunha (Tabela 3).

Os dois tipos de extrato pirolenhoso e as diferentes diluições testadas não tiveram efeito de repelência significativo, pelo teste $\mathrm{F}$, ao nível de $5 \%$ de probabilidade. O número de ácaros na barreira adesiva não ultrapassou $5 \%$, mesmo $48 \mathrm{~h}$ após a aplicação dos tratamentos.

A continuidade da ação do EP de 24 para $48 \mathrm{~h}$ sobre a mortalidade de $B$. phoenicis sugere que pode haver um efeito residual, o que poderia ser estudado utilizando-se da metodologia apropriada para tal finalidade. Se comprovado um efeito residual importante, tal resultado justificaria a diminuição da concentração a ser aplicada. Seria muito importante avaliar os efeitos da aplicação dos diferentes tipos de EP sobre os ácaros em condição de campo, assim como a fitotoxicidade de concentrações maiores que 1:300.

Em função dos resultados obtidos no presente trabalho, pode-se concluir que os dois tipos de extrato pirolenhoso testados não apresentaram repelência significativa sobre Brevipalpus phoenicis; ambos induziram mortalidade significativa somente para concentrações maiores ou iguais a 1:150, com efeito mais pronunciado para o destilado; há um aumento na mortalidade de 24 para 48 horas após a aplicação; a ação protetora preconizada pela aplicação de baixas doses (1:300 a 1:600) nas plantas não é devida à mortalidade e repelência pelo contato direto do extrato pirolenhoso sobre os ácaros.

TABELA 2 - Efeito da interação entre o tipo de extrato pirolenhoso e o tempo após a aplicação na mortalidade $(\%)$ de B. phoenicis $\left(25 \pm 3^{\circ} \mathrm{C}\right.$ e UR 70 $\pm 10 \%)$.

\begin{tabular}{lccc}
\hline \multirow{2}{*}{ Tipo de EP } & \multicolumn{2}{c}{ Tempo após a aplicação } & \multirow{2}{*}{ Teste $\mathrm{F}$} \\
\cline { 2 - 3 } & $24 \mathrm{~h}$ & $48 \mathrm{~h}$ & \\
\hline Destilado & $24,49 \mathrm{Ba}$ & $38,78 \mathrm{Aa}$ & $249,15^{* *}$ \\
Decantado & $11,84 \mathrm{Bb}$ & $18,98 \mathrm{Ab}$ & $62,29^{* *}$ \\
\hline Teste $\mathrm{F}$ & $195,46^{* *}$ & $478,42^{* *}$ & \\
\hline
\end{tabular}

Médias seguidas de mesma letra maiúscula na linha e minúscula na coluna não diferem entre si, pelo teste de Tukey, a $5 \%$ de probabilidade.

** significativo ao nível de $1 \%$ de probabilidade.
TABELA 3 - Efeito da interação entre as diluições de extrato pirolenhoso e o tempo após a aplicação, na mortalidade (\%) de B. phoenicis $\left(25 \pm 3^{\circ} \mathrm{C}\right.$ e UR 70 $\pm 10 \%)$.

\begin{tabular}{|c|c|c|c|}
\hline \multirow{2}{*}{$\begin{array}{l}\text { Proporções } \\
\text { EP: } \mathrm{H}_{2} \mathrm{O}\end{array}$} & \multicolumn{2}{|c|}{ Tempo após a aplicação } & \multirow{2}{*}{ Teste F } \\
\hline & 24 horas & 48 horas & \\
\hline $1: 19$ & $67,14 \mathrm{Ba}$ & $77,14 \mathrm{Aa}$ & $34,88 * *$ \\
\hline $1: 38$ & $30,00 \mathrm{Bb}$ & $66,43 \mathrm{Ab}$ & $462,89 * *$ \\
\hline $1: 75$ & $16,43 \mathrm{Bb}$ & $35,00 \mathrm{Ac}$ & $120,31 * *$ \\
\hline $1: 150$ & $9,29 \mathrm{Bc}$ & 12,86 Ad & $4,45^{*}$ \\
\hline $1: 300$ & $2,86 \mathrm{Bd}$ & 6,43 Ae & $4,45 *$ \\
\hline $1: 600$ & 1,43 Ad & $2,86 \mathrm{Ae}$ & $0,71^{\mathrm{ns}}$ \\
\hline Testemunha & $0,00 \mathrm{Ad}$ & $1,43 \mathrm{Ae}$ & $0,71^{\mathrm{ns}}$ \\
\hline Teste F & $393,64 * *$ & $693,93 * *$ & \\
\hline
\end{tabular}

Médias seguidas de mesma letra maiúscula na linha e minúscula na coluna não diferem entre si, pelo teste de Tukey, a $5 \%$ de probabilidade.

* significativo ao nível de $5 \%$ de probabilidade

** significativo ao nível de $1 \%$ de probabilidade.

${ }^{\mathrm{ns}}$ não-significativo

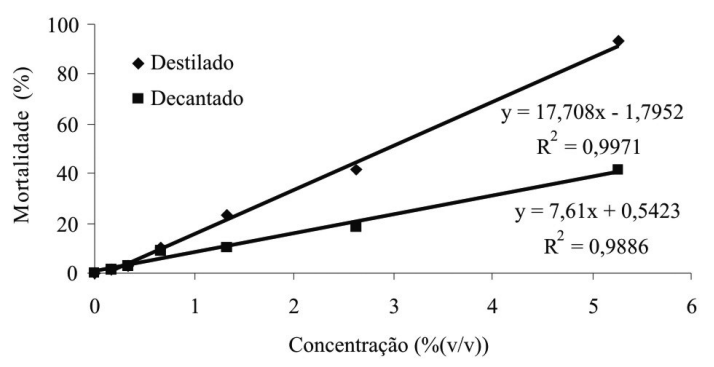

FIGURA 1 - Porcentagem de mortalidade de B. phoenicis $24 \mathrm{~h}$ após a aplicação de concentrações crescentes de extrato pirolenhoso destilado ou decantado $(25 \pm$ $3^{\circ} \mathrm{C}$ e UR $\left.70 \pm 10 \%\right)$.

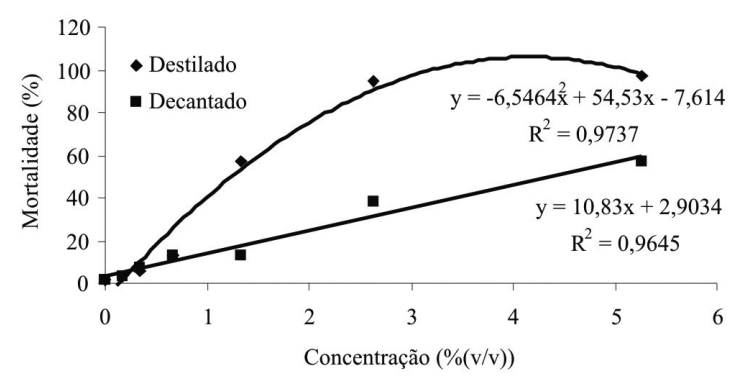

FIGURA 2 - Porcentagem de mortalidade de B. phoenicis $48 \mathrm{~h}$ após a aplicação de concentrações crescentes de extrato pirolenhoso destilado ou decantado $(25 \pm$ $3^{\circ} \mathrm{C}$ e UR $\left.70 \pm 10 \%\right)$. 


\section{REFERÊNCIAS}

ALVES, E.B.; CASARIN, N.F.B.; OMOTO, C. Mecanismos de dispersão de Brevipalpus phoenicis (Geijskes) (Acari: Tenuipalpidae) em pomares de citros. Neotropical Entomology, Curitiba, v.34, n.1, p.89-96, 2005.

CAMPOS NETO, H.H.; MOURA, E.; PASSOS, H.R.; CINIGLIO NETO, F.; MARICONI, F.A.M.; SCARPARI FILHO, J.A. Combate experimental ao ácaro da leprose Brevipalpus phoenicis (Geijskes, 1939) em citros. Scientia Agricola, Piracicaba, v.50, n.2, p. 267-271, 1993.

MAEKAWA, K. Curso sobre produção de carvão, extrato pirolenhoso e seu uso na agricultura. Botucatu: APAN, 2002. mimeografado.

MIYASAKA, S.; OHKAWARA, T.; NAGAI, K.; YAZAKI, H.; SAKITA, M.N. Técnicas de produção e uso do Fino de Carvão e Licor Pirolenhoso. In: ENCONTRO DE PROCESSOS DE PROTEÇÃO DE PLANTAS, 1., 2001, Botucatu. Controle ecológico de pragas e doenças: resumo... Botucatu: APAN, 2001.p.161-176. 